\title{
Performance of a cold storage air-conditioning system using tetrabutylammonium bromide clathrate hydrate slurry
}

\author{
Z.W. Ma, P. Zhang*, R.Z. Wang \\ Institute of Refrigeration and Cryogenics, Shanghai Jiao Tong University, Shanghai 200240, China \\ *Corresponding author. Tel: +86 021 34205505, Fax: +86 021 34206814, E-mail: zhangp@sjtu.edu.cn
}

\begin{abstract}
A cold storage air-conditioning system was built to investigate the energy saving effect of using tetrabutylammonium bromide (TBAB) clathrate hydrate slurry (CHS) as cold storage medium, the corresponding $C O P$ during $\mathrm{TBAB}$ CHS generation and the pumping power during the cold release were measured. As the increase of the TBAB CHS mass fraction during the generation, the system COP generally decreased from about $1.92-2.95$ to about $1.05-1.49$, depending on TBAB CHS flow rate. To clarify the performance of this system, several cold storage strategy cases are studied, the corresponding electric power consumption and the cost saving, compared with water as the cold transportation medium, are shown in this work as well.
\end{abstract}

Keywords: Clathrate hydrate slurry, Secondary loop air-conditioning system, evaluation.

\section{Introduction}

As a $\mathrm{n}$ ew kind of phase change slurry material, tetrabutylammonium bromide $\left(\left[\mathrm{CH}_{3}\left(\mathrm{CH}_{2}\right)_{3}\right]_{4} \mathrm{NBr}\right.$, TBAB in abbreviate) clathrate hydrate slurry (CHS) was studied by many researchers in recent years. Due to the adjustable phase change temperature over the range of $5-12{ }^{\circ} \mathrm{C}$, the good cold-carry capacity which is about 2-4 times of that of chilled water and the good fluidity, TBAB CHS is considered promising in an air-conditioning system, where this slurry can be used both as cold storage and transportation medium.

Researchers in Japan firstly reported this new material for air-conditioning using, and measured the basic thermo-physical properties, including the phase diagram, latent heat, density, heat capacity, thermal conductivity etc. [1,2], and they also applied it to a real application [3]. Hayashi et al. [1], Darbouret et al. [4], Xiao et al. [5] and Ma et al. [6] all investigated the flow characteristics of TBAB CHS in straight tubes, nevertheless, the results reported were divergent. Moreover, Ma et al. [6] reported the forced convective heat transfer characteristics. However, the application of TBAB CHS is still limited by the deficient studies, and the performance in an air-conditioning system is rarely reported.

In the present study, a cold storage air-conditioning system using TBAB CHS was built and the corresponding performance was presented. System COP during TBAB CHS generation and the pumping power during cold release were both measured, based on which the system energy consumption was numerically estimated in different cases. In addition, the cost saving of using TBAB CHS compared with that of using chilled water as the cold transportation medium was evaluated.

\section{Methodology}

\subsection{Basic thermo-physical properties of TBAB CHS}

The TBAB CHS is a kind of solid-liquid suspension with white color, as shown in Fig. 1, which can be easily generated at atmosphere condition by cooling down the TBAB aqueous solution to supercooling state. The melting temperature, mass fraction, latent heat and other properties (such as density, heat capacity and thermal conductivity) of TBAB CHS are introduced in this section. 


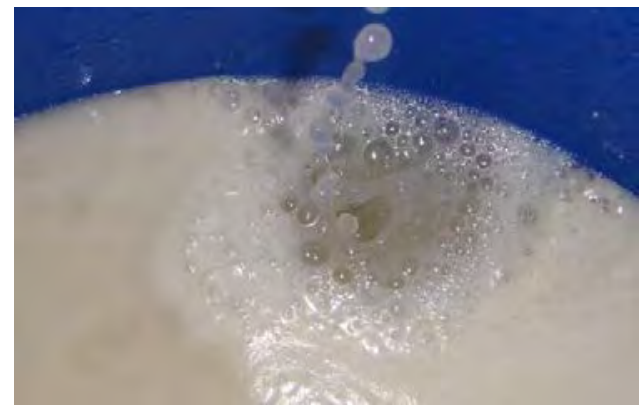

Fig. 1. Photo of the TBAB CHS.

The melting temperature could be determined with the aid of the phase diagram, which describes the relation between it and the corresponding solution concentration. Differential Scanning Calorimeter (DSC, TA, Q2000) was applied to record the heating processes of TBAB CHS at a heating rate of $0.5^{\circ} \mathrm{C} / \mathrm{min}$, and then the phase diagram could be plotted based on the test results, which is shown in Fig. 2 as well as the comparison with that obtained by other researchers.

The volume and mass fraction of CHS can be determined by Eq. (1) and (2), with several requisite parameters provided by the phase diagram.

$\omega_{\mathrm{p}}=\frac{\omega_{0}-\omega_{\text {liq }}}{\omega_{\mathrm{H}}-\omega_{\text {liq }}}$

$\varphi=\frac{\omega_{\mathrm{p}} / \rho_{\mathrm{p}}}{\omega_{\mathrm{p}} / \rho_{\mathrm{p}}+\left(1-\omega_{\mathrm{p}}\right) / \rho_{\text {liq }}}$

where $\omega_{\mathrm{p}}$ is the crystal mass fraction of TBAB CHS, $\omega_{0}$ is the initial solution concentration, $\omega_{\mathrm{H}}$ is the TBAB mass fraction in the hydrate crystal, $\omega_{\text {liq }}$ is the concentration of the liquid phase in the slurry, $\varphi$ is the TBAB CHS volume fraction, $\rho_{\mathrm{p}}$ and $\rho_{\text {liq }}$ are densities of the crystal and the liquid phase, respectively. Two different hydrates with different hydration numbers were observed, and the corresponding thermo-physical properties have been summarized by Ma et al. [6], as shown in Table 1, the two values of some properties in the table were the result of different researches referenced.

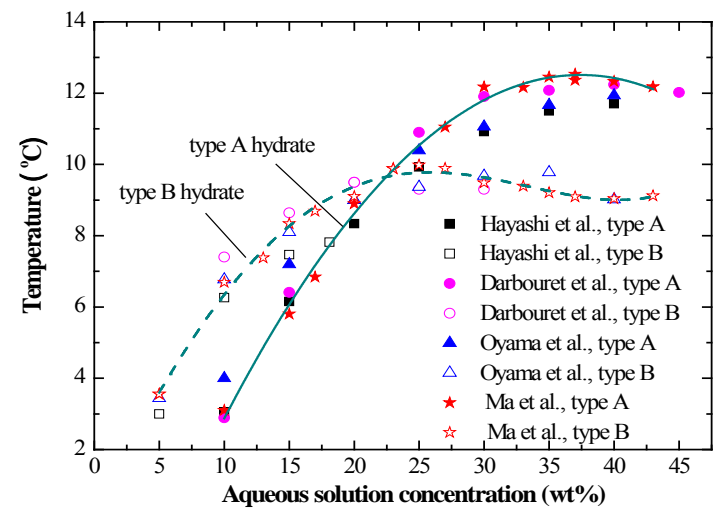

Fig. 2. Phase diagram of TBAB CHS. [6] 
Table 1. Thermo-physical properties of TBAB hydrate crystals [6].

\begin{tabular}{ccccccc}
\hline & $\begin{array}{l}\text { Hydration } \\
\text { number } \\
-\end{array}$ & $\begin{array}{l}\text { Melting } \\
\text { temperature } \\
\left({ }^{\circ} \mathrm{C}\right)\end{array}$ & $\begin{array}{l}\text { Density } \\
\left(\mathrm{kg} / \mathrm{m}^{3}\right)\end{array}$ & $\begin{array}{l}\text { Latent heat } \\
(\mathrm{kJ} / \mathrm{kg})\end{array}$ & $\begin{array}{c}\text { Heat } \\
\text { capacity } \\
(\mathrm{kJ} /(\mathrm{kg} \cdot \mathrm{K}))\end{array}$ & $\begin{array}{l}\text { Thermal } \\
\text { conductivity } \\
(\mathrm{W} /(\mathrm{m} \cdot \mathrm{K}))\end{array}$ \\
\hline type A & 26 & $11.8 / 12.0$ & 1080 & $193.18 \pm 8.52$ & $\begin{array}{c}2.22 / 1.86- \\
2.61\end{array}$ & 0.42 \\
type B & $36 / 38$ & 9.90 & 1030 & $199.59 \pm 5.28$ & $2.00-2.54$ & - \\
\hline
\end{tabular}

Density of TBAB aqueous solution was measured using a balance (FS: 2200g, accuracy: 0.01 g) and a graduated flask (FS: $50 \mathrm{~mL}$, accuracy: $1 \mathrm{~mL}$ ), while the heat capacity was measured based on the heat balance with water in a plate heat exchanger. Thereafter, these properties of TBAB CHS can be calculated by the corresponding values of TBAB solution we measured and that of TBAB hydrate crystal given in Table 1. M eanwhile, thermal conductivity of TBAB aqueous solution as well as TBAB CHS was measured by a transient hot-wire unit (the measuring error was less than $\pm 3 \%$ while water was applied). All the properties of TBAB CHS (type B hydrate, original $15 \mathrm{wt} \%$ solution) are presented in Table 2.

Table 2. Thermo-physical properties of TBAB CHS (type B hydrate crystal, original 15 wt\% solution).

\begin{tabular}{cccc}
\hline $\begin{array}{c}\text { Mass } \\
\text { fraction } \\
(\mathrm{wt} \%)\end{array}$ & $\begin{array}{c}\text { Density } \\
\left(\mathrm{kg} / \mathrm{m}^{3}\right)\end{array}$ & $\begin{array}{c}\text { Heat } \\
\text { capacity } \\
(\mathrm{kJ} /(\mathrm{kg} \cdot \mathrm{K}))\end{array}$ & $\begin{array}{c}\text { Thermal } \\
\text { conductivity } \\
(\mathrm{W} /(\mathrm{m} \cdot \mathrm{K}))\end{array}$ \\
\hline 5 & 1015.690 & 4.001 & 0.469 \\
10 & 1015.656 & 3.933 & 0.473 \\
15 & 1015.635 & 3.865 & 0.476 \\
20 & 1015.608 & 3.798 & 0.480 \\
25 & 1015.585 & 3.732 & 0.483 \\
30 & 1015.560 & 3.667 & 0.485 \\
\hline
\end{tabular}

The enthalpy change $(Q)$ of TBAB CHS in a certain temperature range, $5-12{ }^{\circ} \mathrm{C}$ for example, is an important parameter which indicates how much cold energy is stored or released. Basically, there are two methods to calculate the enthalpy change of this slurry. One is introducing the slurry fraction change $(\Delta \omega)$, the latent heat $(\Delta H)$ and the sensible heat $(C p \Delta T)$ into Eq. (3):

$Q=\Delta \omega \Delta H / 100+C p \Delta T$

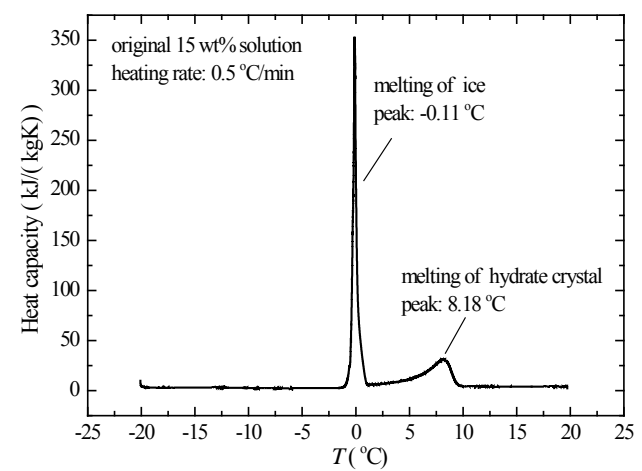

(a)

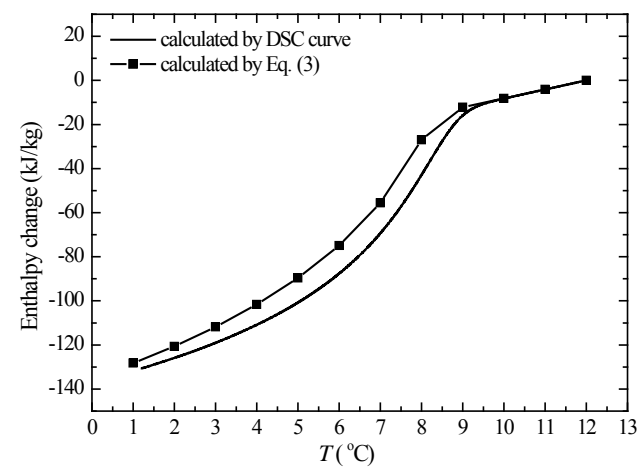

(b)

Fig. 3. (a) DSC curve; (b) enthalpy change of TBAB CHS. 
The other method is integrating the DSC heating curve against temperature shown in Fig. 3(a). The comparison between these two methods is shown in Fig. 3(b). The enthalpy change within the temperature range of 5 to $12{ }^{\circ} \mathrm{C}$ calculated by Eq. (3) is about $10.9 \%$ smaller than that calculated by DSC curve, and the latter method was applied in the present work.

\subsection{Experimental set-up and CHS generation method}

Fig. 4 shows the schematic diagram of the constructed cold storage air-conditioning system using TBAB CHS. Two thermal insulated tanks $\left(1.2 \mathrm{~m}^{3}\right)$ were used for solution and slurry, respectively. Three Pt100 sensors (accuracy: $\pm 0.15{ }^{\circ} \mathrm{C}$ ) were employed to record the temperature variations at the bottom, middle and top of the each tank. A double-tube heat exchanger (ShenShi, GT-U0480) with corrugated flow passage was applied to undertake the heat exchange between solution and the evaporating refrigerant. The refrigerator used was an outdoor unit of a commercial air-conditioner, which can switch from cooling to heating by a four-way valve. The used slurry pump was a speed adjustable rotational pump and a stabilizing tank located at the downstream of the pump was used to stabilize the flow. A simple agitator was mounted on the slurry tank, which was operated to avoid the deposition of crystals. A plate heat exchanger (Swep, B8 $\times 30$ ) was selected as the load side heat exchanger since its high heat transfer rate, and hot water acted as the cooling load. The hot water was stored at an insulated water tank before experiment and was drained after used. Moreover, pressure sensors (accuracy: $0.1 \%$ ) were located at different positions of the fluid flow to measure the pressure drop, and the electric power consumed by the refrigerator and the pump were measured as well.

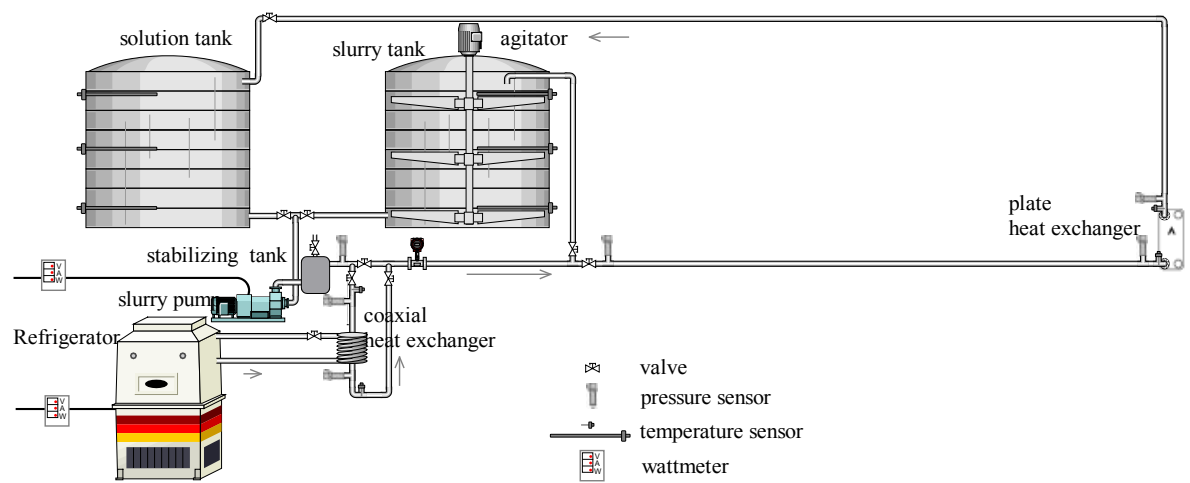

Fig. 4. Schematic diagram of the experimental set-up.

The test was divided into three steps based on the state of the TBAB CHS: (1) The aqueous solution was cooled to supercooling state. However, the supercooling state cannot be achieved by only one cycle from the solution tank to the slurry tank due to the limited cooling power of the refrigerator. As a consequence, the solution must be continuously pumped through the double-tube heat exchanger from and back to the slurry tank. (2) Hydrate crystals appeared. Accompanied with mechanical shocks between the returned fluid and the stored fluid, TBAB hydrate crystals can be generated in the supercooled solution, and the fluid temperature increased because of the released latent heat. Moreover, sometimes the agitator should be operated to accelerate the hydrate crystal generation. (3) TBAB CHS was kept cooled to reach the desired crystal fraction. (4) TBAB CHS was pumped to the load side to release the stored cold energy, and afterwards became aqueous solution again and flowed back to the solution tank.

The crucial disadvantage during the entire test occurred in step 3. Before the desired hydrate fraction was achieved, TBAB CHS was continuously cooled and hydrate crystals grown 
inside the heat exchanger. The generated crystals would adhere to the heat transfer surface where the temperature was extraordinary low. The adhered crystals layer deteriorated the heat transfer between refrigerant and TBAB CHS. The worse thing was that the refrigerant temperature dropped a lot to maintain the heat exchange, which resulted in forming more hydrate crystals and creating thicker crystal layer on the heat transfer surface. A malignant cycle occurred, the hydrate crystals were difficult to be continuously produced and the system efficiency became low.

Three methods are mainly proposed to solve the aforementioned low-efficiency problem: (1) Maintaining the refrigerant temperature at a certain temperature range by adjusting the refrigerant flow rate. (2) Increasing the flow velocity of the TBAB CHS to be high enough to flush and break off the crystal formed on the flow passage wall so that to ensure a good heat transfer. (3) Operate the refrigerator reversely from cooling to heating for a while to melt the adhered hydrate crystals. In this work, a manual needle throttle valve was applied to implement the first method, and it was found that more accurate control of the throttle valve was needed. Thus, the method (2) and (3) were applied to ensure the continuous generation of hydrate crystals and high system efficiency, as shown in Fig. 5. With high flow rate (about 16-18 kg/min in Fig. 5) and reverse operation, we obtained $31 \mathrm{wt} \%$ TBAB CHS successfully.

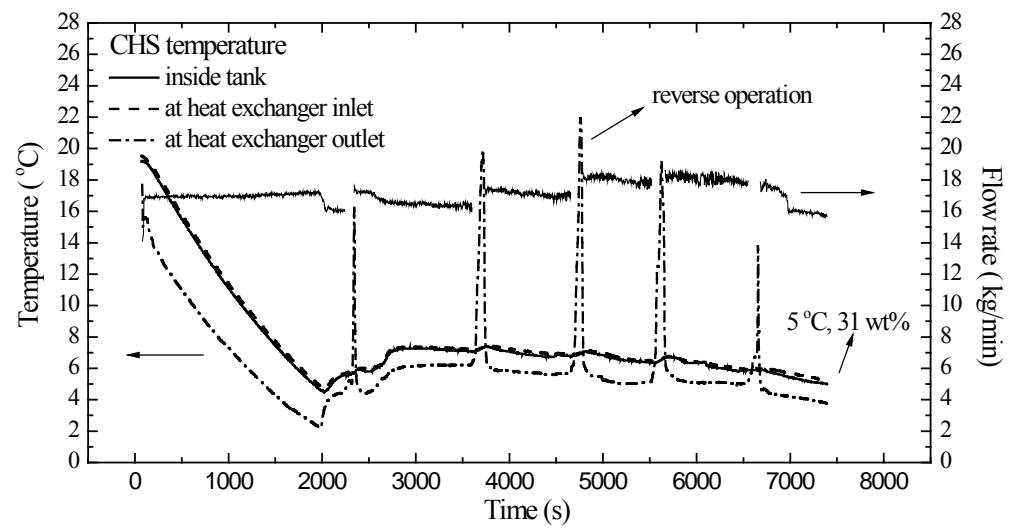

Fig. 5. Typical generation process of TBAB CHS.

\section{Results and discussions}

The system COP during the CHS generation was calculated by Eq. (4):

$C O P=\frac{\text { cold energy stored by TBAB CHS }}{\text { refrigerator power }+ \text { pumping power }}$

It should be claimed that the stored cold energy was all calculated from $15{ }^{\circ} \mathrm{C}$ solution to the stored TBAB CHS. Fig. 6 shows COP as function of the mass fraction as well as the flow rate. The COP of $0 \mathrm{wt} \%$ TBAB CHS shown in the figure was the average system COP before the hydrate appearance. The hydrate crystals were generated instantaneously, thereafter about 14$16 \mathrm{wt} \%$ mass fraction was soon reached, hence COP from the beginning to this moment was higher than that before the generation. However, as increase of the mass fraction, COP reduced from about 1.92-2.95 to about 1.05-1.49 due to the aforementioned crystals adherence to the heat transfer surface. Meanwhile, the reverse operation of refrigerator consumed additional energy, which was another attributor to the COP reduction. As mentioned, high flow velocity was beneficial to the heat transfer between TBAB CHS and refrigerant, since the adhered crystals would be flushed down by the strong shear force, which 
was validated by COP profile shown in the figure - higher flow rate generally led to a higher $C O P$. However, this phenomenon depended on the performance of the pump.

The pumping power during the cold release will be reduced if TBAB CHS is applied as the secondary refrigerant instead of chilled water due to its higher cold-carry capacity and thus the flow rate is lower. Fig. 7 presents the pumping power of water, $20 \mathrm{wt} \% \mathrm{CHS}, 25 \mathrm{wt} \%$ CHS and $30 \mathrm{wt} \%$ CHS as the function of the cooling load during the cold release. As seen in the figure, more energy saving on pumping power was achieved by using TBAB CHS with higher mass fraction.
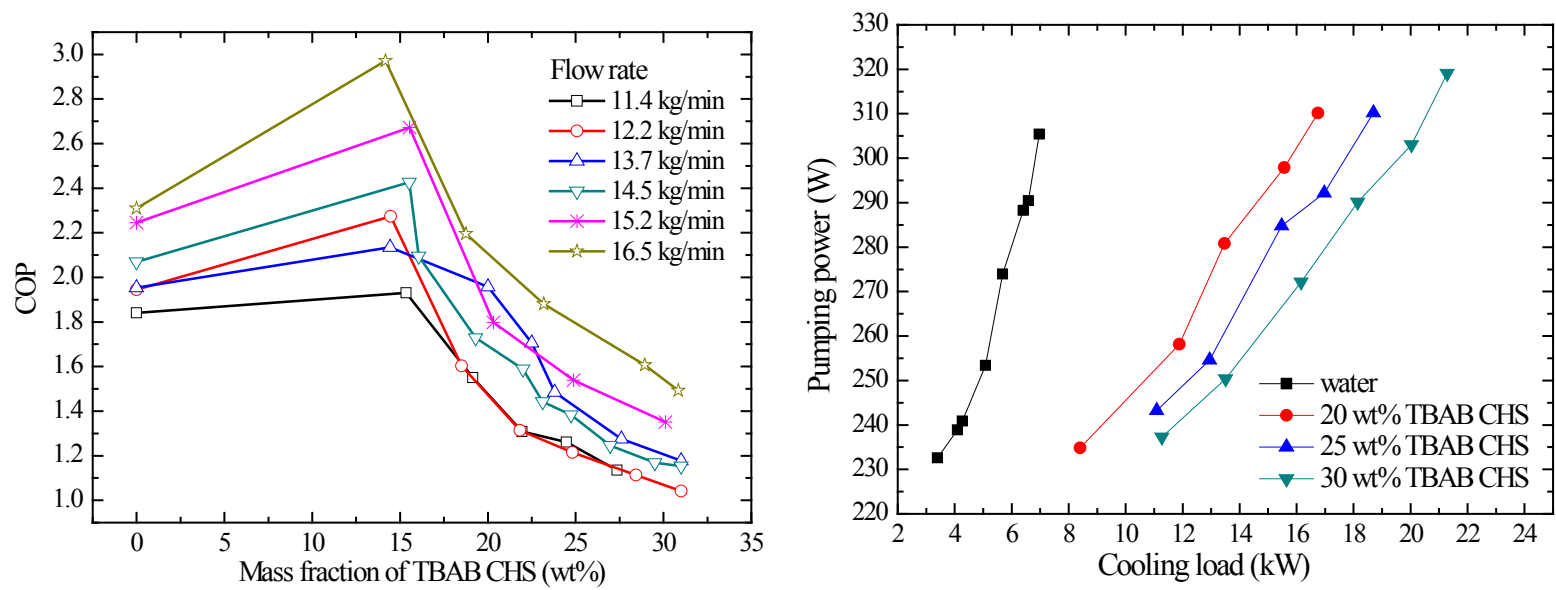

Fig. 6. System COP during the CHS generation. Fig. 7. Pumping power during the cold release.

A study case is assumed for better exhibiting the performance of TBAB CHS. The assumed cooling load is in eight hours in daytime, during which the average cooling load is $21 \mathrm{~kW}$ and the maximum value is $33 \mathrm{~kW}$, therefore the totally cooling duty is $604800 \mathrm{~kJ}$. Fig. 8 shows four types of system operation strategies. For each case, $20 \mathrm{wt} \%, 25 \mathrm{wt} \%$ and $30 \mathrm{wt} \% \mathrm{CHS}$ are applied, the system COP during the TBAB CHS generation is about 2.10, 1.79 and 1.54 , respectively based on the results in Fig. 6 (flow rate: $16.5 \mathrm{~kg} / \mathrm{min}$ ). The storage ratio (which is the ratio of the storage cold energy to the total required cold energy) of case 1 and case 2 is $40 \%$ while that of case 3 and case 4 is $60 \%$, the other cooling load is satisfied by the refrigerator (average system COP is about 2.32) while considering water as the secondary refrigerant. Moreover, assume the application of water as case $5 \mathrm{f}$ or the comparison, and consider water as the secondary refrigerant for all the cold release and no cold storage is conducted.

Fig. 9(a) shows the electric power consumption with all the study cases based on the present system. It can be seen from the figures, the power consumption increases as the increase of the mass fraction, which is obviously caused by the lower system COP. All the power consumptions in cases 1-4 are higher than that of case 5, which means there is no energy saving of the application of TBAB CHS compared with water. However, the operation cost does decrease as the increase of TBAB CHS mass fraction and the cost saving is shown in Fig. 9(b) (the price of the electricity is taken as $0.3 \mathrm{RMB} / \mathrm{kWh}$ during night time while 0.6 during daytime), about $8-27 \%$ cost saving can be achieved. However, since the the present system is limited by the room space, the piping from the storage tank to the load side is very short and thus the pumping power shown in Fig.7 is not coincident to the practical system with the assumed cooling load. Therefore, we re-calculate all the cases with amplifying the pumping power to 3 times as large as the present measured values during the cold release, the original case 1-5 become to case $1^{\prime}-5$ '. Fig. 10(a) and (b) show the corresponding electric power 
consumption and the cost saving. It is noticed that about $1.4-3.5 \%$ energy saving is achieved, calculated by $20 \mathrm{wt} \%$ TBAB CHS with all the operation strategies, while the cost saving increases to about $10-29 \%$.
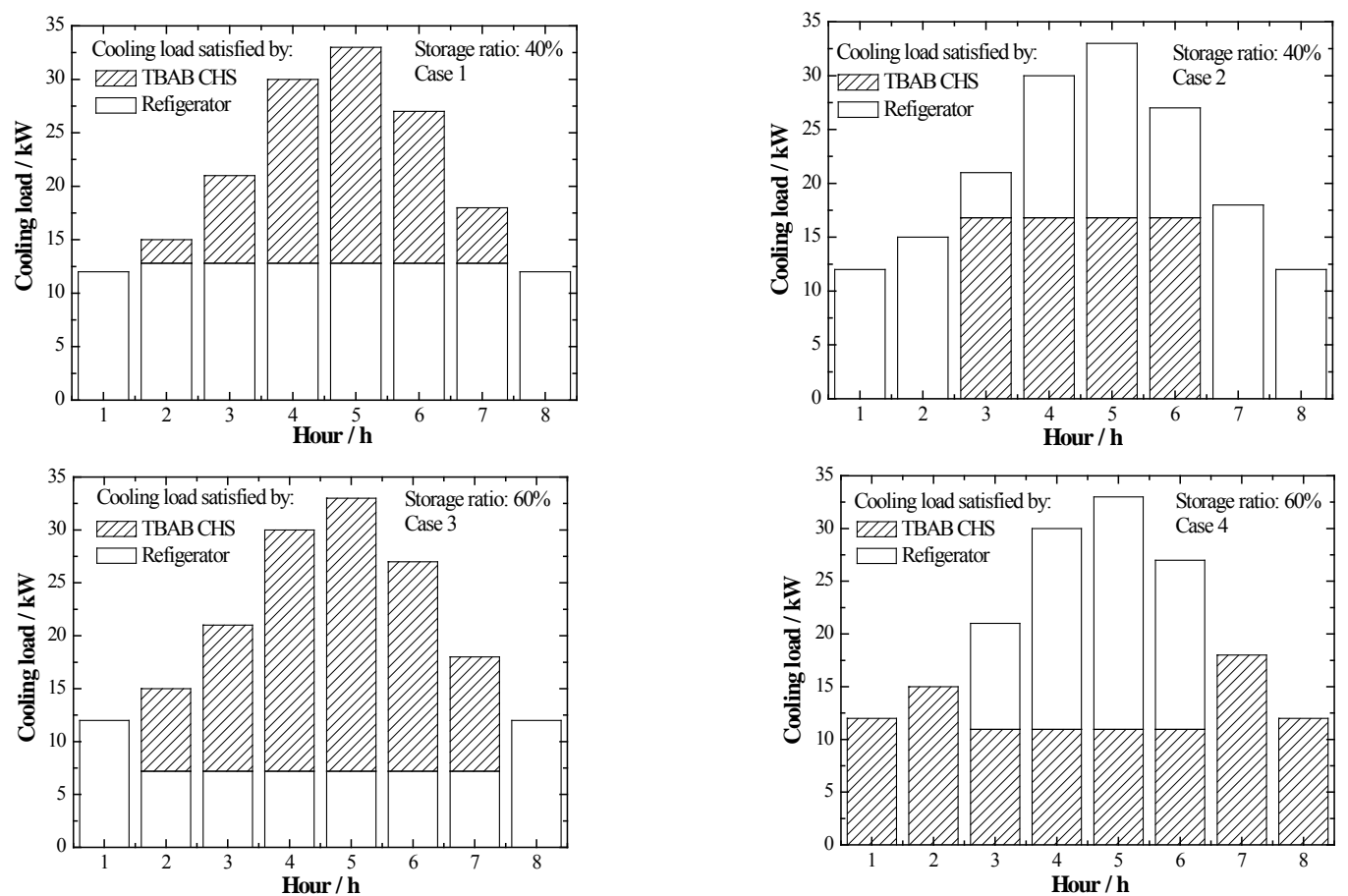

Fig. 8. System operation strategies.

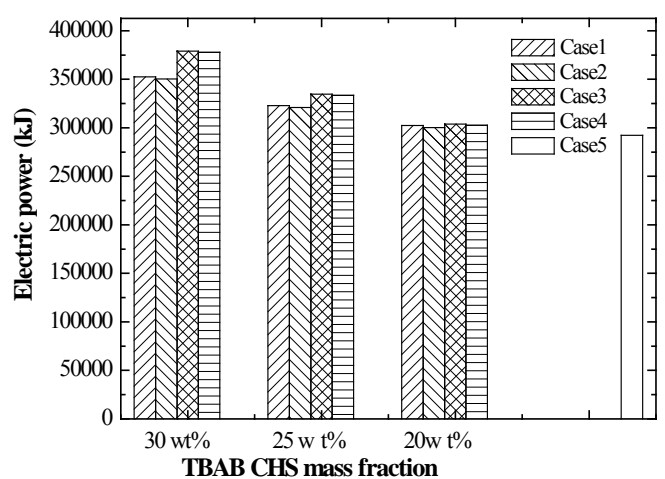

(a)

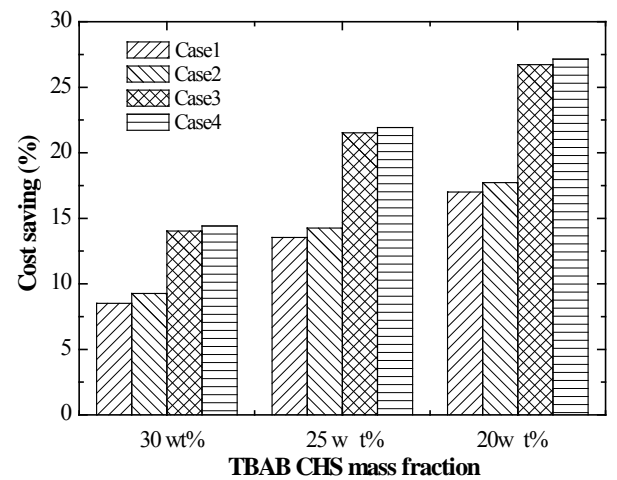

(b)

Fig. 9. Electric power consumption and cost saving.

\section{Conclusion}

The present work mainly constructed and tested a cold storage air-conditioning system using TBAB CHS and estimates the energy consumption. The system COP decreased from about 1.92-2.95 to about 1.05-1.49 during CHS generation. The energy saving by using TBAB CHS instead of water was not achieved as expected since the piping was short and the pumping power was low in the present system, while $8-27 \%$ cost saving was achieved. However, about $1.4-3.5 \%$ energy saving could be achieved if the pumping power was amplified to 3 times as large as the original values, meanwhile the cost saving was about 10 $29 \%$. 


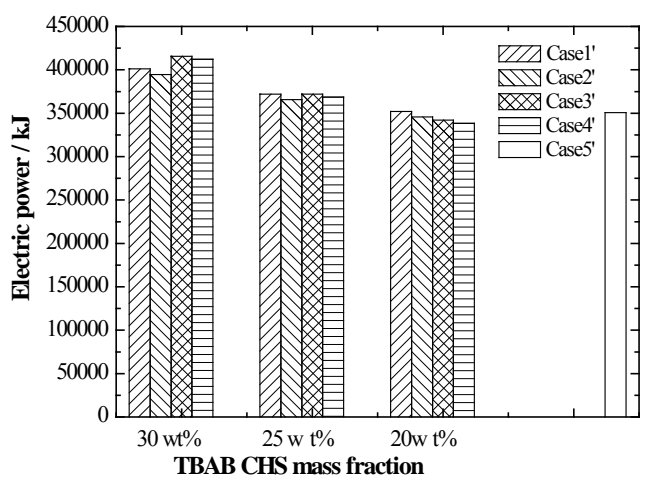

(a)

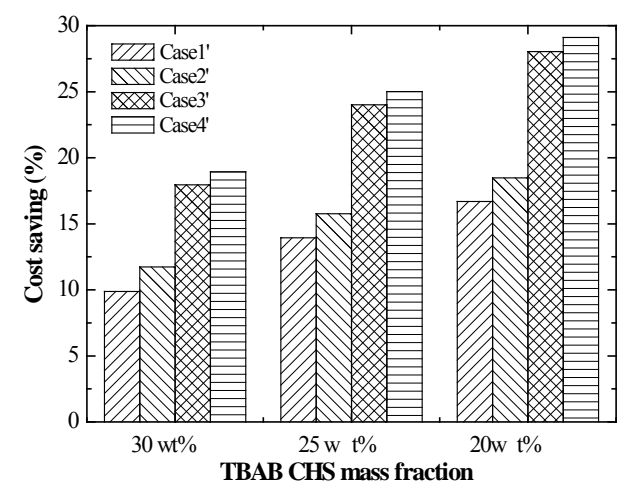

(b)

Fig. 10. Electric power consumption and cost saving with amplified pumping power.

\section{Acknowledgements}

The authors are grateful to the "Shu Guang" project supported by Shanghai Municipal Education Commission and Shanghai Education Development Foundation under the Contract No. 09SG11 and the Science and Technology Commission of Shanghai Municipality under the Contract No. 10160710700. This research is also partially supported by the Specialized Research Fund for the Doctoral Program of Higher Education under the Contract No. 20100073110039.

\section{References}

[1] K. Hayashi, S. Takao, H. Ogoshi and S. Matsumoto, Research and development on highdensity cold latent-heat medium transportation technology, IEA Annex-10-PCMs and Chemical Reactions for Thermal Energy Storage 4th Workshop, Japan, 2000.

[2] H. Oyama, W. Shimada, T. Ebinuma, Y. Kamata, S. Takeya, T. Uchida, J. Nagao and H. Narita, Phase diagram, latent heat, and specific heat of TBAB semiclathrate hydrate crystals, Fluid Phase Equilibria 234, 2005, pp. 131-135.

[3] S. Takao, H. Ogoshi, S. Matsumoto, K. Takashi, M. Sugiyama, T. Akiyama, S. Fukushima, New air conditioning systems using hydrate slurry, NKK Technical Report 174, 2001, pp. 6-11. [in Japanese]

[4] M. Darbouret, M. Cournil, and J.M. Herri, Rheological study of TBAB hydrate slurries as secondary two-phase refrigerants, International Journal of Refrigeration 28, 2005, pp. 663-671.

[5] R. Xiao, S.S. Wu, L.G. Tang, C. Huang, and Z.P. Feng, Experimental investigation of the pressure-drop of clathrate hydrate slurry (CHS) flow of tetra butyl ammonium bromide (TBAB) in straight pipe, Proceedings of $10^{\text {th }}$ International Conference on $\mathrm{T}$ hermal Energy Storage, Stockton, USA, 2006.

[6] Z.W. Ma, P. Zhang, R.Z. Wang, S. Furui, and G.N. Xi, Forced flow and convective melting heat transfer of clathrate hydrate slurry in tubes, International Journal of Heat and Mass Transfer 53, 2010, pp. 3745-3757. 